\title{
Matter-Dominated Muon Accelerator Lattice Simulation Tools for COSY Infinity
}

\author{
J. Kunz ${ }^{1}$, P. Snopok ${ }^{1}$, M. Berz ${ }^{2}$, and K. Makino ${ }^{2}$ \\ ${ }^{1 .}$ Illinois Institute of Technology, Department of Physics, Chicago, IL, United States. \\ 2. Michigan State University, Department of Physics and Astronomy, East Lansing, MI, United States.
}

A prime example of why matter-dominated lattices are relevant comes from the prospect of a neutrino factory or a muon collider [1]. As muon branching fractions are nearly $100 \% \mu^{-} \rightarrow e^{-} \bar{v}_{e} v_{\mu}$ and $\mu^{+} \rightarrow$ $e^{+} v_{e} \bar{v}_{\mu}$, there are obvious advantages of a muon-sourced neutrino beam. Also, due to the fact that muons are roughly 200 times heavier than electrons, synchrotron radiation is not an issue, and as a result a high-energy muon collider $(\sqrt{\mathrm{s}}=6 \mathrm{TeV})$ could be built on a relatively compact site (where the collider ring is about $6 \mathrm{~km}$ in circumference). This energy level is experimentally unprecedented in the leptonic sector, since a circular electron accelerator would be restricted by vast amounts of synchrotron radiation. At lower energy, a muon collider could serve as a Higgs factory $(\sqrt{\mathrm{s}}=126 \mathrm{GeV})$, with possible new physics via the observation of Higgs to lepton coupling. This is advantageous since the Higgs theoretically couples more strongly to muons than electrons because of the small electron mass.

However, muon-based facilities are not without their challenges. Synthetic muon creation comes from the collision of protons with a fixed target. The resultant spray of particles largely contains kaons (which decay primarily into pions and muons), pions (which decay primarily into muons), and rogue protons. High-intensity collection necessarily entails a large initial phase space volume. The resultant cloud of muons must be collected, focused, and accelerated well within the muon lifetime ( $2.2 \mu s$ at rest). Therefore, beam cooling (beam size reduction) techniques which are commonly used for protons and electrons cannot be used, as they are too slow. Due to the short-lived nature of the muon, novel beam cooling techniques have been explored and ionization cooling in particular has been shown to work quite well [2]. Here, muons traverse a certain amount of material in order to lose energy in both longitudinal and transverse directions due to ionization. The energy is then restored in the longitudinal direction only by passing through a set of RF cavities, leading to an overall reduction in the transverse direction (cooling). Schematically, this can be seen in Figure 1, where this process is split into three parts.

COSY Infinity (COSY) [3,4] is a simulation tool used in the design, analysis, and optimization of particle accelerators, spectrographs, beam lines, electron microscopes, and other such devices, with its use in accelerator lattice design being of particular interest here. COSY uses the transfer map approach, in which the overall effect of the optics on a beam of particles is evaluated using differential algebra. Along with tracking of particles through a lattice, COSY has a plethora of analysis and optimization tools, including computation of Twiss parameters, tunes and nonlinear tune shifts, high-order nonlinearities; analysis of properties of repetitive motion via chromaticities, normal form analysis, and symplectic tracking; analysis of single-pass systems resolutions, reconstructive aberration correction, and consideration of detector errors; built-in local and global optimizers; and analysis of spin dynamics.

COSY is particularly advantageous to use when considering the efficient use of computational time. This is due to the transfer map methods that COSY employs. Given an initial phase space vector $\mathbf{Z}_{\mathbf{0}}$ at $\mathbf{s}_{0}$ that describes the relative position of a particle with respect to the reference particle, and assuming the future evolution of the system is uniquely determined by $\mathbf{Z}_{\mathbf{0}}$, we can define a function called the transfer map relating the initial conditions at $\mathrm{s}_{0}$ to the conditions at $\mathrm{s}$ via $\mathbf{Z}(\mathrm{s})=\boldsymbol{\mathcal { M }}\left(\mathrm{s}_{0}, \mathrm{~s}\right) * \mathbf{Z}\left(\mathrm{s}_{0}\right)$. The transfer 
map formally summarizes the entire action of the system. The composition of two maps yields another map: $\boldsymbol{M}\left(\mathrm{s}_{0}, \mathrm{~s}_{1}\right) \circ \boldsymbol{\mathcal { M }}\left(\mathrm{s}_{1}, \mathrm{~s}_{2}\right)=\boldsymbol{\mathcal { M }}\left(\mathrm{s}_{0}, \mathrm{~s}_{2}\right)$, which means that transfer maps of systems can be built up from the transfer maps of the pieces. Computationally this is advantageous because once calculated, it is much faster to apply a single transfer map to a distribution of particles than to track individual particles through multiple lattice elements.
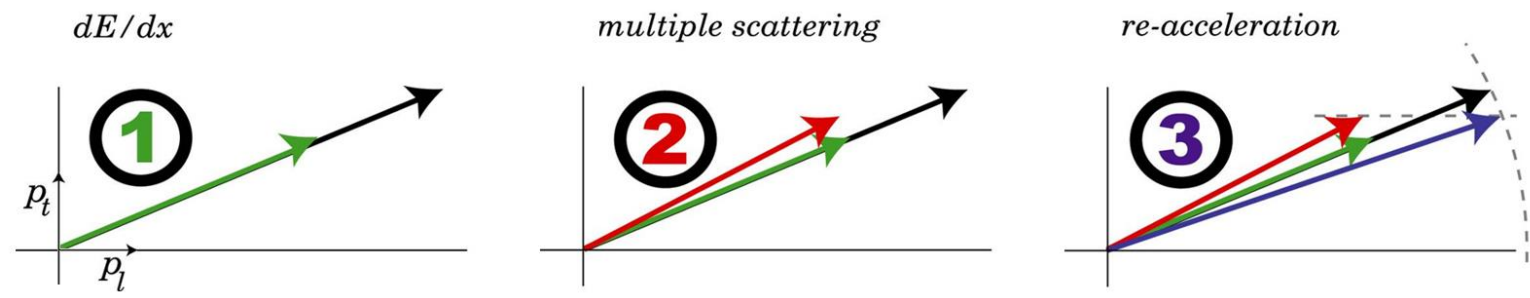

Figure 1. Vector representation of ionization cooling. 1) Energy loss in material, both transverse and longitudinal momenta are reduced. 2) Increase in the transverse momentum due to multiple scattering. 3) Re-acceleration through the RF cavity resulting in the net reduction in the transverse momentum.

Currently supported elements in COSY include various magnetic and electric multipoles (with fringe effects), homogeneous and inhomogeneous bending elements, Wien filters, wigglers and undulators, cavities, cylindrical electromagnetic lenses, general particle optical elements, and deterministic absorbers of intricate shapes described by polynomials of arbitrary order, with the last element being of particular interest for this study. The term deterministic is deliberately emphasized, since the polynomial absorber acts like a drift with the average (Bethe-Bloch) energy loss. The advantage of this is that the user must only specify six material parameters in order for COSY to calculate this energy loss: the atomic number, atomic mass, density, ionization potential, and two correction parameters.

However, this element only takes into account deterministic effects (producing the same final result every time for the same initial condition), not stochastic effects (intrinsically random effects such as multiple scattering and energy straggling). For a realistic simulation of a beam of particles through matter, one needs to take into account both the former and the latter. The evolution of the normalized transverse emittance can be described by the following equation:

$$
\frac{d \epsilon_{n}}{d z} \approx-\frac{1}{\beta^{2}}\left\langle\frac{d E_{\mu}}{d z}\right\rangle \frac{\epsilon_{n}}{E_{\mu}}+\frac{1}{\beta^{3}} \frac{\beta_{\perp} E_{s}^{2}}{2 E_{\mu} m c^{2} X_{0}},
$$

where $\epsilon_{\mathrm{n}}$ is the normalized emittance, $\mathrm{z}$ is the path length, $E_{\mu}$ is the muon beam energy, $\beta=\mathrm{v} / \mathrm{c}, \mathrm{X}_{0}$ is the radiation length of the absorber material, $\beta_{\perp}$ is the betatron function, and $E_{S}$ is the characteristic scattering energy [5]. Here, two competing effects can be seen: the first term is the cooling (reduction of phase space beam size) component from ionization energy loss and the second term is the heating (increase of phase space beam size) term from multiple scattering. This highlights the importance of the stochastic terms, as the only deterministic term is the expected (Bethe-Bloch) energy loss, $\left\langle d E_{\mu} / d z\right\rangle$.

It is clear why stochastic effects do not fit well into the transfer map paradigm: two identical particles with identical initial coordinates may follow two different paths inside the absorber material due to the intrinsically random nature of multiple scattering. Therefore, it is not possible to construct a traditional transfer map that represents the absorber, as this would require uniquely relating the coordinates after the absorber to the coordinates before the absorber. In light of this, the effort of integrating stochastic 
processes into COSY focuses on particle-by-particle propagation (as opposed to transfer map methods). Furthermore, in the spirit of efficiency this integration should endeavor to greatly increase the step size of such algorithms, and to include other possible improvements.

The typical treatment of multiple scattering involves three steps: free propagation, angular correction, and lateral displacement correction, as depicted by Figure 2. As previously discussed, Figure 2 shows only one of many possibilities. The angular correction and lateral displacement are chosen from probability distributions, which vary by material, absorber length, and initial energy. Figure 3 clearly shows the dependence of the lateral correction on absorber length corresponding to longitudinal momentum losses of 5, 10, 15, and $20 \mathrm{MeV} / \mathrm{c}$. Since the initial distribution is a pencil beam (i.e. $x=p_{x}=\sigma_{x}=\sigma_{p_{x}}=0$ ), Figure 3 shows virtually all of the different possible final states for $10^{4}$ muons with the same initial conditions. From here the probability distribution of the lateral displacement correction can be ascertained as roughly Gaussian, where the Gaussian mean is always zero (i.e. the most probable scenario is no net scattering) and the Gaussian $\sigma$ appears to be some function of absorber length, and possibly absorber material and initial energy.

\section{Actual Scattering}

\section{$\underline{\text { Simulation }}$}

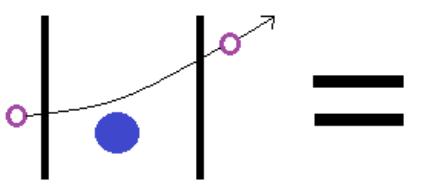

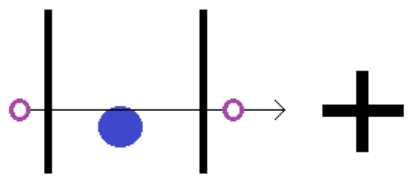

1. Propagation

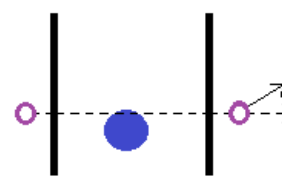

2. Angular Correction

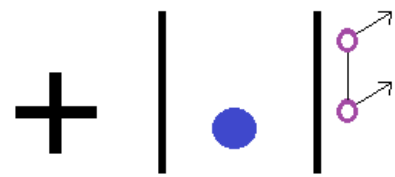

3. Lateral Correction

Figure 2. Left: a depiction of classical scattering. Right: an algorithm producing a similar effect.
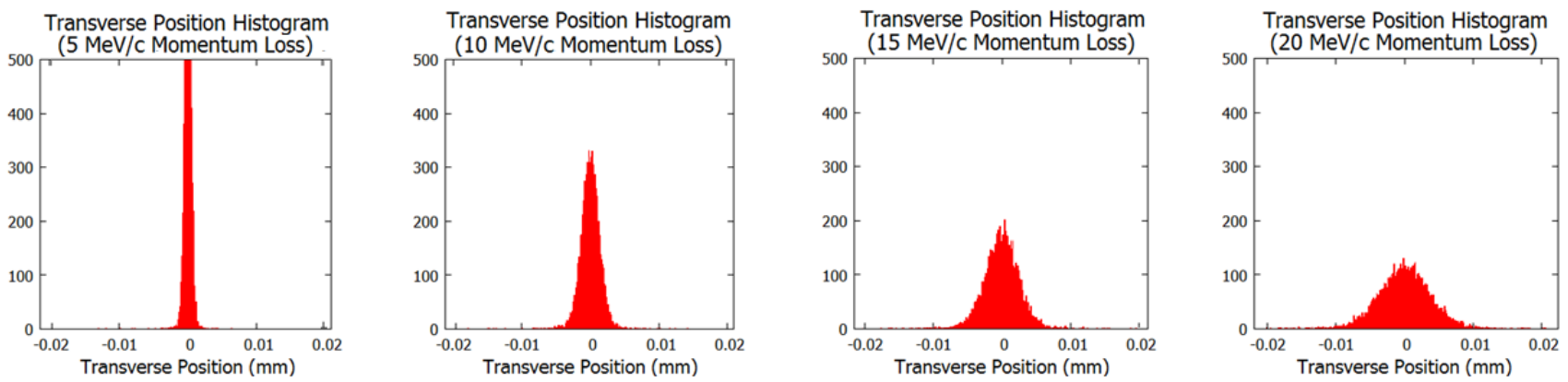

Figure 3. $10^{4}$ muons were simulated with ICOOL [6] through various lengths of liquid hydrogen. These transverse position histograms were obtained from a pencil beam with an initial momentum of precisely $200 \mathrm{MeV} / \mathrm{c}$.

More precisely, the distribution should follow Goudsmit-Saunderson theory [7] for small angles and have a Rutherford distribution for large angles. Then the (unnormalized) distribution that represents the angular correction can be described by Eq. (2), which takes into account continuity and smoothness:

$$
g(u)=\left\{\begin{array}{cc}
e^{-a(1-u)} & u_{0} \leq u \quad\left(P_{x} \leq P_{x_{0}}\right) \\
\frac{4 e^{-3}}{(a-1-a u)^{2}} & u \leq u_{0}\left(P_{x_{0}} \leq P_{x}\right)
\end{array}\right.
$$


where $u=\cos (\theta)=P_{z} / \sqrt{P_{z}^{2}+P_{x}^{2}}, u_{0}=1-3 / a$, and $P_{x_{0}}=P_{z} \sqrt{(a /(a-3))^{2}-1}$. Here the Gaussian profile mentioned earlier is recovered for small $P_{x}$, as $1 / \sqrt{1+\left(P_{x} / P_{z}\right)^{2}} \approx 1-0.5\left(P_{x} / P_{z}\right)^{2}$.

The scattering profile is now in terms of the variable $a$. According to modified Highland-Lynch-Dahl theory, it is possible to write: $a\left(L, E_{i}\right)=0.5 /\left(1-\cos \left(\theta_{0}\left(L, E_{i}\right)\right)\right.$, where $\theta_{0}\left(L, E_{i}\right)=13.6 \mathrm{MeV}$ * $L^{0.555} /\left(\beta p c X_{0}^{0.555}\right)$ has been slightly modified [8]. Here, $\beta=v / c, p$ is the total initial momentum, $L$ is the so-called "true path length" (here approximated simply as the step size), and $X_{0}$ is the radiation length of the material. Finally, it is possible to explicitly see the dependence of the scattering distribution on the absorber material $\left(X_{0}\right)$, absorber length $(L)$, and initial energy $(\beta c p)$.

In a similar fashion, straggling (fluctuation about a mean energy loss) may be simulated. The energy loss profile follows a Landau distribution [9] of the form

$$
f(\lambda)=\frac{1}{\xi} * \frac{1}{2 \pi i} \int_{c+i \infty}^{c-i \infty} \exp (u \ln u+\lambda u) d u,
$$

where $c \geq 0$ and $\lambda=(\epsilon-\bar{\epsilon}) / \xi-1+\gamma_{E u l e r}-\beta^{2}-\ln \left(\xi / E_{\max }\right)$. Here $\epsilon$ is the energy loss variable, $\bar{\epsilon}$ is the average energy loss (which is already calculated by baseline COSY), $\gamma_{\text {Euler }}=0.577 \ldots$, and $E_{\max }$ and $\xi$ are defined as

$$
E_{\max }=\frac{2 m_{e} \beta^{2} \gamma^{2}}{1+2 \gamma m_{e} / m+\left(m_{e} / m\right)^{2}}, \quad \xi=\frac{2 \pi z_{c h}^{2} e^{4} N_{A} Z \rho}{A m_{e} c^{2}} * \frac{L}{1-m^{2} / E_{i}^{2}} .
$$

Here $m_{e}$ is the mass of the electron, $\gamma=1 / \sqrt{1-\beta^{2}}, m$ is the mass of the incident particle, $e$ is the fundamental charge, $N_{A}$ is Avogadro's number, $Z$ is the nucleic charge of the material, $\rho$ is the material density, $A$ is the atomic number of the material, $L$ is the length of the absorber, and $E_{i}$ is the incident energy of the particle.

For implementation reasons, it is more helpful to use the Landau function where the energy loss is parameterized as $\lambda=(\epsilon-\alpha) / \beta_{L}$, where $\alpha$ is approximately the most probable value and $\beta_{L}$ is a "scaling factor" referred to as the Landau beta. By comparison with the former parameterization, it is easy to infer that $\beta=\xi$ and that

$$
\alpha\left(L, E_{i}\right)=\bar{\epsilon}+\beta_{L}\left(L, E_{i}\right)\left[2-\gamma_{\text {Euler }}-\frac{m^{2}}{E_{i}^{2}}+\ln \left(\frac{\beta_{L}\left(L, E_{i}\right)}{E_{\max }}\right)\right] .
$$

Eqns. (2) and (3) (with the variables $a, \beta_{L}$, and $\alpha$ as functions of material, absorber length, and initial energy) were implemented into COSY as post-absorber corrections. Figures 4 , and 5 show the results of these implementations compared to ICOOL [6] and G4Beamline [10], where ICOOL uses the default setting for straggling (Vavilov straggling) and Bethe-modified Molière scattering. The initial beam parameters for this simulation were $5 \times 10^{4}$ muons through $12 \mathrm{~mm}$ of liquid hydrogen (left) and liquid helium (right) with $\left(x, \sigma_{x}, P_{x}, \sigma_{P_{x}}, \sigma_{P_{z}}\right)=\overrightarrow{0}$, where $x$ signifies the arbitrary transverse direction, and $P_{z}=200 \mathrm{MeV} / \mathrm{c}$. 

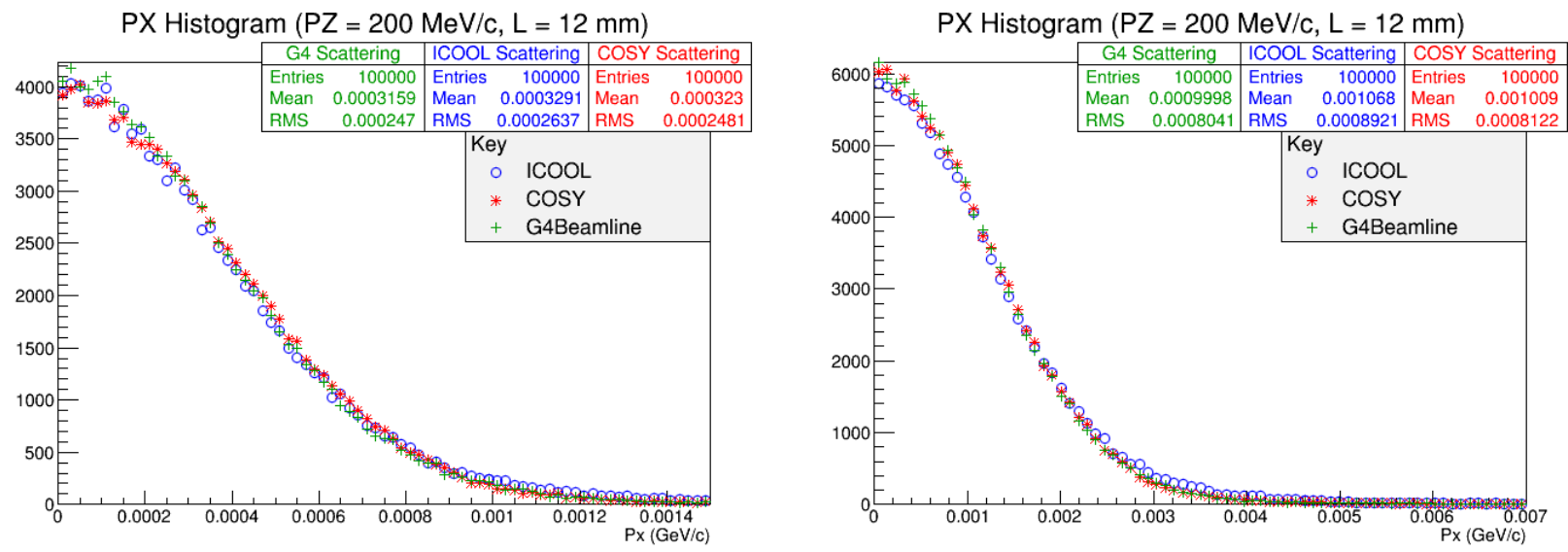

Figure 4. $\left|P_{x}\right|$ histogram comparison between COSY Infinity, ICOOL, and G4Beamline showing good agreement between the codes. Left is liquid hydrogen, right is lithium hydride.
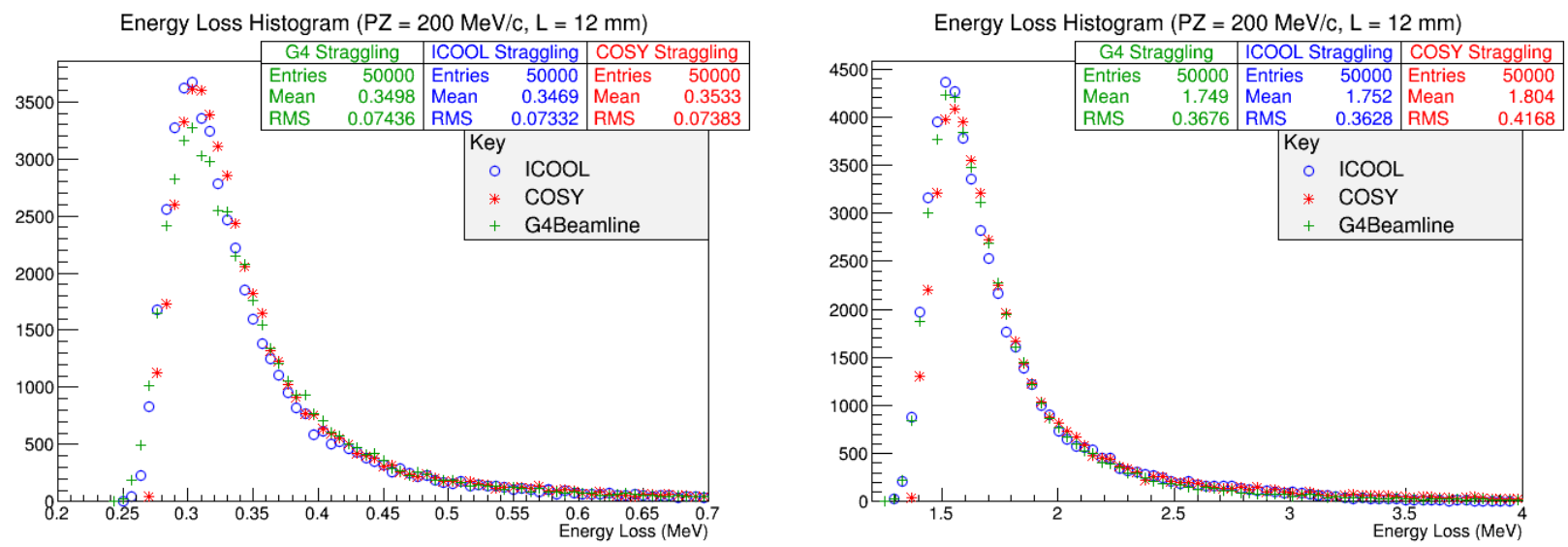

Figure 5. Energy loss histogram comparison between COSY Infinity, ICOOL, and G4Beamline showing good agreement between the codes. Left is liquid hydrogen, right is lithium hydride.

Based on the current results, future work should include several improvements. The most obvious improvement is to increase the step size even further: up to, for example, $10 \mathrm{~cm}$. For scattering, it is presently unclear how this increase will affect the theoretical curves. On the other hand, when considering energy loss effects theory predicts a Vavilov distribution [11] of the form

$$
f(\epsilon)=\frac{1}{\xi} \frac{1}{2 \pi i} \int_{c-i \infty}^{c+i \infty} \exp \left[\kappa\left(1+\beta^{2} \gamma\right)+\psi(s)+\lambda s\right] d s, \quad c \geq 0,
$$

where

$$
\psi(s)=s \ln \kappa+\left(s+\beta^{2} \kappa\right)\left[\ln (s / \kappa)+\int_{\infty}^{s / \kappa} t^{-1} e^{-t} d t\right]-\kappa e^{-s / \kappa}
$$

and $\kappa\left(L, E_{i}\right)=\xi / E_{\max }$. Recall that $E_{\max }$ and $\xi$ can be found in Eqn. (4). $\lambda_{v}$ can be shown to be related to the Landau $\lambda$ as $\lambda=\lambda_{v} / \kappa-\ln \kappa$. Moreover, as $\kappa \rightarrow 0$ the Vavilov distribution tends to the Landau 
distribution, and as $\kappa \rightarrow \infty$ the Vavilov distribution tends to a Gaussian distribution. For computational efficiency it is common to set these limits as

$$
f(\epsilon)=\left\{\begin{array}{lr}
\text { Landau, } & \kappa \leq 0.01 \\
\text { Vavilov }, & 0.01 \leq \kappa \leq 10 \\
\text { Gauss }, & 10 \leq \kappa
\end{array}\right.
$$

More implementations into this code consider very small step sizes $(\sim 0.1 \mathrm{~mm})$ for the purpose of propagation of the beam through absorbers inside of high magnetic fields. For any realistic simulation of muons, decay processes must be included as well. This naturally leads to the inclusion in the code of daughter particle tracking. Finally, the algorithms presented here should be subject to comparison with experimental results, such as the Muon Scattering Experiment [12].

In summary, muon-based neutrino sources are enticing due to their ability to create consistent, highintensity mixed neutrino beams. On the other hand, muon colliders present an opportunity to conduct leptonic experiments at unprecedented energy levels while keeping the facility size quite compact. However, for both of these facilities ionization cooling is a crucial component. The design of these novel cooling channels requires many simulation software tools to be augmented with new experimental results for various types of absorbers. COSY Infinity has many features for advanced lattice design, and will in the future be outfitted with tools that can accurately evaluate matter-dominated lattices of a wide variety. One of these tools will parameterize probability distribution function terms in order to add a stochastic perturbative kick at the end of a step inside such a lattice, which will emulate random effects. These step sizes will be able to be varied without significant loss of accuracy from $0.1 \mathrm{~mm}$ (for high magnetic fields) to $10 \mathrm{~cm}$ (for no magnetic fields).

\section{References:}

[1] Fermilab, "Muon Accelerator Program," (2014), map.fnal.gov.

[2] V Parkhomchuk and A Skrinsky, 12th Int. Conf. on High Energy Accel. (1983), p. 485

[3] M Berz and K Makino, "COSY Infinity," (2014), www.cosyinfinity.org.

[4] M Berz in "Modern Map Methods in Particle Beam Physics", (Academic Press, London).

[5] D Neuffer, Part. Acc. 14 (1983), p. 75.

[6] RC Fernow, "ICOOL Simulation Code", (2013), https://pubweb.bnl.gov/ fernow/icool.

[7] S Goudsmit and JL Saunderson, Phys. Rev. 57 (1940), p. 24.

[8] GR Lynch and O Dahl, Nucl. Instrum. Meth. B 58 (1975), p. 6.

[9] L Landau, J. Phys.-USSR 8 (1944), p. 201.

[10] T Roberts, “G4Beamline”, (2013), http://www.muonsinternal.com/muons3/G4beamline.

[11] PV Vavilov, Sov. Phys. JETP-USSR 5 (1957), p. 749.

[12] D Attwood et al, Nucl. Instrum. Meth. B 251 (2006), p. 41. 